\title{
Experimental Demonstration of a Squeezing-Enhanced Power-Recycled Michelson Interferometer for Gravitational Wave Detection
}

\author{
Kirk McKenzie, Daniel A. Shaddock, and David E. McClelland \\ Gravitational Wave Research Facility, Department of Physics, Faculty of Science, Australian National University, \\ ACT 0200, Australia \\ Ben C. Buchler and Ping Koy Lam \\ Quantum Optics Group, Department of Physics, Faculty of Science, Australian National University, ACT 0200, Australia
} (Received 14 February 2002; published 23 May 2002)

\begin{abstract}
Interferometric gravitational wave detectors are expected to be limited by shot noise at some frequencies. We experimentally demonstrate that a power recycled Michelson with squeezed light injected into the dark port can overcome this limit. An improvement in the signal-to-noise ratio of $2.3 \mathrm{~dB}$ is measured and locked stably for long periods of time. The configuration, control, and signal readout of our experiment are compatible with current gravitational wave detector designs. We consider the application of our system to long baseline interferometer designs such as LIGO.
\end{abstract}

DOI: $10.1103 /$ PhysRevLett.88.231102

The first generation of interferometric gravitational wave $(\mathrm{GW})$ detectors are expected to begin taking data in 2002. Although they will be the most sensitive devices ever built, they are predicted to detect only large, infrequent gravitational events. To regularly detect sources, and thereby allow comparison with astrophysical models, a factor of 10 improvement in sensitivity is required. The second generation of detectors, such as Advanced LIGO [1], is expected to reach this goal. These instruments will employ $100 \mathrm{~W}$ class lasers, light recycling techniques, fused silica suspension systems, and high mechanical $Q$ sapphire mirror substrates. Early predictions are that they will be limited by quantum noise, i.e., noise arising from the quantum nature of light, over most of the GW signal frequency band (10 to $1000 \mathrm{~Hz})$ [1]. This has sparked an explosion of theoretical proposals on the application of quantum optical techniques to surpass the quantum limit in laser interferometry. These include the following: the use of squeezed states [2] building on the proposal by Caves [3]; quantum nondemolition schemes including changing of the readout parameters [4] and optomechanical coupling techniques [5]. What makes these theoretical proposals even more exciting is the maturity of experimental techniques for generating nonclassical states of light. In 1985, the landmark experiment of Slusher et al. [6] measured $0.3 \mathrm{~dB}$ of quantum noise suppression. Benchtop experiments currently produce over $7 \mathrm{~dB}$ reduction of the quantum noise $[7,8]$. A combination of current squeezing technology with the high power and high stability of GW detection laser and optical systems now makes $10 \mathrm{~dB}$ of squeezing a realistic goal [2].

Despite the potential for squeezing to improve interferometric sensitivity, until now there has been no experimental demonstration of squeezing applied to an interferometer bearing any resemblance to a GW detector. Squeezingenhanced performance has been demonstrated in other interferometers, such as the Mach-Zehnder [9] and po-
PACS numbers: 04.80.Nn, 07.60.Ly, 42.50.Dv, 42.50.Lc

larimeter [10]. None of these experiments employed a Michelson configuration, used light recycling techniques; or utilized a signal readout scheme compatible with an advanced GW detector. Theoretical analysis of GeaBanacloche et al. [11] suggested that squeezing is broadly compatible with recycling techniques. However, the difficulty in devising a readout and control scheme compatible with both squeezing and light recycling has, until now, prevented any definitive demonstration.

In this Letter, we report an experimental demonstration of a power-recycled Michelson [12] with locked optical squeezing injected into the dark port. Our source of squeezing was an optical parametric amplifier (OPA). We used a laser system, configuration, control and readout system compatible with advanced GW detector proposals. The entire system maintained lock for long periods, and we measured a signal with noise below the shot noise limit (SNL). Contrary to previous work, we found that there is a link between power recycling and squeezing. In fact, the efficiency of squeezing detected at the dark port with power recycling can be higher than for a simple Michelson. Given the success of our experiment, we review theoretically the compatibility and application of our system to long baseline detectors, such as LIGO [13].

The experimental setup is shown in Fig. 1. Of the $700 \mathrm{~mW}$ of power generated by our laser (Nd:YAG $1064 \mathrm{~nm}$ ), about $70 \%$ was used to pump the squeezing system. The remainder passed through a mode-cleaning cavity [14], giving an output beam which was shot-noise limited above $4 \mathrm{MHz}$. This beam provided both the interferometer input and a seed wave for the OPA. The low power $(\sim 10 \mu \mathrm{W})$ squeezed output of the OPA was phase modulated at $15.8 \mathrm{MHz}$. Details of the OPA system may be found in Ref. [15]. The squeezed light was injected into the interferometer via a Faraday rotator (Gsänger FR 1060/5) to give spatial degeneracy between the injected squeezing and the output beam of the interferometer, 


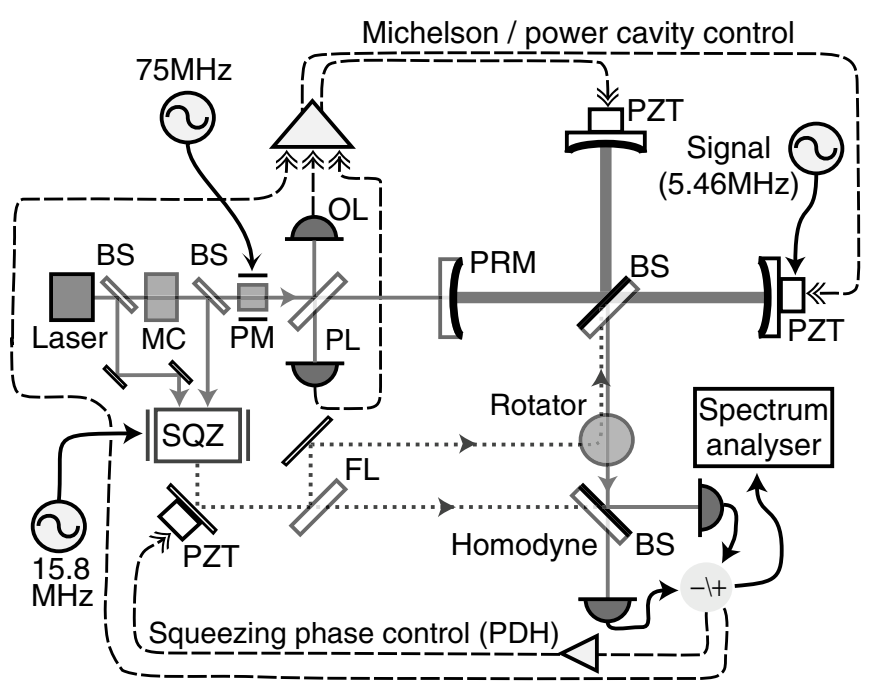

FIG. 1. Schematic of the experiment. Squeezed light can be injected into the output port of the interferometer via the Faraday rotator or interrogated using homodyne detection. The control servos for the interferometer are shown schematically by the dashed lines. $\mathrm{BS}=$ beam splitter; $\mathrm{CL}=$ power cavity locking detector; $\mathrm{FL}=$ flipper mirror; $\mathrm{OL}=$ offset locking detector; $\mathrm{PM}=$ phase modulator $(75 \mathrm{MHz})$; $\mathrm{PZT}=$ piezoelectric transducer; and $\mathrm{SQZ}=$ squeezed state generation system.

as required in a long baseline $\mathrm{GW}$ detector. The fringe visibility between the squeezing and the interferometer input beam was $99 \%$. The squeezed beam could also be measured directly by the homodyne system via a flipper mirror. The interferometer had a power cavity of length $1 \mathrm{~m}$ and power mirror of $90 \%$ reflectivity. The input beam to the interferometer had a power of $20 \mathrm{~mW}$, of which $3 \mathrm{~mW}$ exited at the dark port where it was measured by the homodyne system. The homodyne detectors were built around ETX 500 photodiodes with 93\% quantum efficiency. A GW signal was simulated by modulating one of the Michelson arm lengths using a piezoelectric transducer (PZT) at a frequency outside the power cavity bandwidth. In our case this was $5.46 \mathrm{MHz}$ [16]. The signal readout was obtained from the sum output of the homodyne detectors.

A vital feature of our setup is that the control scheme is compatible with future GW detectors and squeezed light. For squeezing to be of any use, the squeezed quadrature must be in phase with the interferometer output. The 15.8 $\mathrm{MHz}$ phase modulation of the squeezed beam allowed us to use the Pound-Drever-Hall (PDH) [17] method to gather an error signal for the squeezed beam phase. This was fed back to a PZT in the squeezed beam path. The power cavity error signal was also derived using the PDH technique (using the $75 \mathrm{MHz}$ phase modulation) and was fed back to the length of the power cavity, via PZTs on the Michelson mirrors. The relative length of the Michelson arms was controlled with offset locking. This error signal was derived by subtracting an offset voltage, obtained via the offset locking detector, from the sum voltage of the homodyne detectors [18]. The error signal was fed back to PZTs on the Michelson mirrors. Offset locking is being used in Advanced LIGO prototypes [19] and is highly compatible with squeezing. It requires no modulation so that, unlike Schnupp/frontal modulation [20], the signal readout does not require demodulation. This is a benefit as the squeezing is required only at the GW signal frequencies. If modulation techniques are used, squeezing is required at the signal frequency and twice the modulation frequency \pm the signal frequency [21]. This simplification of the requirements for squeezing in a $\mathrm{GW}$ detector is valuable.

Squeezed states of light may also be used to suppress radiation pressure noise in GW detectors by tuning the angle of the squeezed quadrature $[2,22]$. Our experimental setup can, in principle, be used to suppress radiation pressure noise; however, our experiment does not operate in a regime where radiation pressure is significant. Suppression of radiation pressure noise is, therefore, not considered in this Letter.

Shot noise arises as the electromagnetic vacuum mode enters the dark port of the beam splitter. The vacuum mode interferes with the input beam passing on its noise characteristics. The smallest phase a SNL interferometer can measure is

$$
\Delta \phi \geq 1 / \sqrt{n},
$$

where $n$ is the number of photons incident on the beam splitter. Subshot noise sensitivity can be achieved if the vacuum mode is filled by a squeezed state. The noise suppression can be characterized by the intensity variance of the light at the interferometer output, $V_{\mathrm{pd}}$ (where the variance is normalized to the SNL). When optimized, this variance is a function of the input beam intensity noise, $V_{\mathrm{LO}}$, the squeezed state noise, $V_{\mathrm{sqz}}$, and the losses in the interferometer, modeled by adding vacuum noise with variance $V_{v}=1$. The normalized transfer functions for each noise input to the photodetector for our system are $T_{\mathrm{LO}}(\omega), T_{\mathrm{sqz}}(\omega)$, and $T_{v}(\omega)$, so that the detected variance $V_{\mathrm{pd}}$ is given by

$$
V_{\mathrm{pd}}=\left|T_{\mathrm{LO}}(\omega)\right|^{2} V_{\mathrm{LO}}+\left|T_{\mathrm{sqz}}(\omega)\right|^{2} V_{\mathrm{sqz}}+\left|T_{v}(\omega)\right|^{2} V_{v}
$$

These transfer functions are frequency independent for a simple Michelson. If a power recycling mirror is present, they are functions of the power recycling cavity linewidth. When the interferometer is locked close to a dark fringe, the intensity fluctuations on the input beam couple weakly to the interferometer output; i.e., $T_{\mathrm{LO}}$ is very small. The amount of squeezing that couples to the output, however, is large as $T_{\mathrm{sqz}}(\omega)$ is close to 1 . This is the dominant term in Eq. (2). To model our experiment, we consider $3 \mathrm{~dB}$ of input squeezing over the detection frequency range. Theoretical plots of the frequency spectra of the detected output variance for a simple Michelson and a power recycled Michelson (PRM) are shown in Fig. 2. The results show improved performance of the PRM compared to the simple Michelson. This difference is a result of two effects. First, to keep the same power at the homodyne photodetector, 


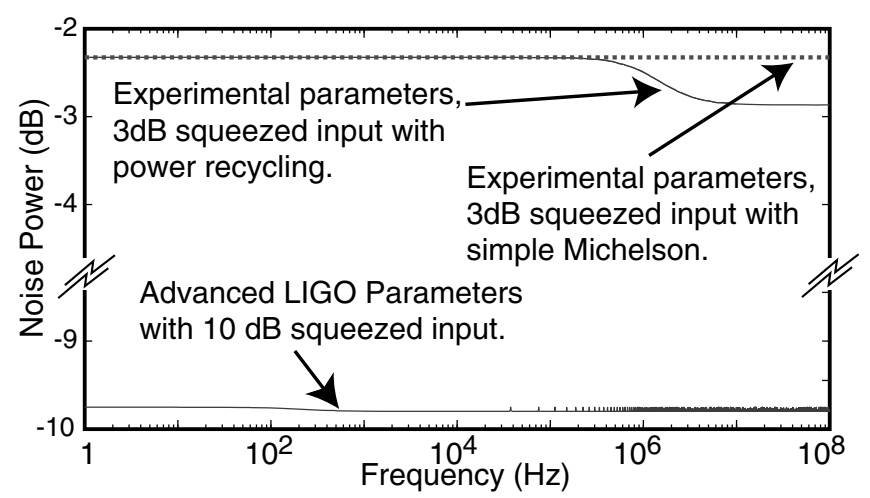

FIG. 2. Predicted shot noise suppression at the dark port is shown as a function of frequency. The vertical scale is $\mathrm{dB}$ relative to the SNL (at $0 \mathrm{~dB}$ ). Top traces: a model of our experiment assuming $3 \mathrm{~dB}$ of squeezing, power mirror reflectivity of $90 \%$ and $15 \%$ rotator loss. Bottom trace: Predicted performance with Advanced LIGO parameters, $10 \mathrm{~dB}$ of squeezing and $5 \%$ rotator loss.

the position of the Michelson fringe shifts, such that the effective Michelson reflectivity increases. In our experiment, the reflectivity for the simple Michelson is $\approx 0.92$, whereas it is $\approx 0.99$ for the PRM. The result is an increase in $T_{\mathrm{sqz}}(\omega)$ and a decrease in $T_{\mathrm{LO}}(\omega)$, so that more squeezing is transferred to the interferometer output. In the presence of a power recycling mirror, therefore, less squeezing is wasted in the interferometer. Second, the power mirror introduces a frequency dependence to the squeezing transfer function, $T_{\mathrm{sqz}}$. Outside the power cavity linewidth, the interferometer becomes highly reflective so that transfer of the squeezing becomes close to ideal.

Figure 3 shows the noise of our squeezed beam prior to entering the interferometer. The noise suppression is $\sim 3.5 \mathrm{~dB}$ below the SNL. When the squeezing was injected into the interferometer, our Faraday rotator gave $15 \%$ loss (double pass). This was the dominant source of loss in our experiment. It reduced the amount of squeezing coupled into the interferometer to $\sim 2.8 \mathrm{~dB}$ (measured).

A signal at $5.46 \mathrm{MHz}$ was used to characterize our Michelson interferometer. Figure 4(a) shows the result for

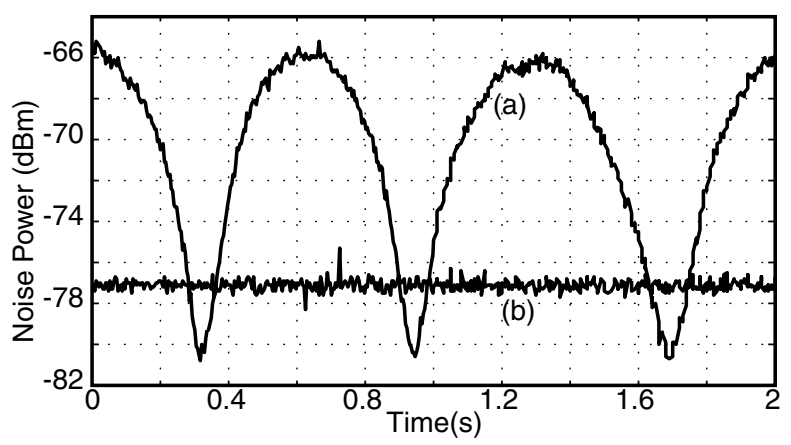

FIG. 3. (a) Scan of squeezing at $5.5 \mathrm{MHz}$ measured before the interferometer by homodyning. (b) Shot noise at $5.5 \mathrm{MHz}$. Electronic noise lies at $-84.9 \mathrm{dBm}$; resolution bandwidth $(\mathrm{RBW})=100 \mathrm{kHz}$; video bandwidth $(\mathrm{VBW})=300 \mathrm{~Hz}$. a simple Michelson. The noise floor is at the SNL. When squeezing was introduced [trace 4(b)], we observed noise suppression of $1.8 \pm 0.2 \mathrm{~dB}$ below the SNL. Traces 4(c) and 4(d) show the response of the PRM with and without the squeezed input. The signal power is proportional to the circulating power. Our power recycling factor of $\approx 4$ therefore gives the PRM a signal $\approx 4$ times larger than the simple Michelson. The noise floor of trace 4(d) is $2.3 \pm 0.2 \mathrm{~dB}$ lower than SNL. The predicted improvement of the squeezing performance with power recycling is evident in the different noise floors of traces 4(b) and 4(d). If the electronic noise is subtracted from trace (d), the squeezed noise floor is found to lie $3.0 \pm 0.2 \mathrm{~dB}$ below SNL. Our experiment maintained lock for periods longer than $15 \mathrm{~min}$, and was limited only by the temperature stability of our laboratory.

These results confirm that squeezing is compatible with power recycling; however, the practicality of using squeezed light in existing and future GW detectors depends on the compatibility with current designs and the amount of squeezing that will be actually available. We will briefly look at these two issues.

In terms of compatibility to proposed GW detectors, there are three outstanding considerations: the requirement of arm cavities, signal recycling, and the generation of squeezing in the GW signal band. The arm cavities introduce fractionally higher loss into the interferometer $(\sim 1 \%$ [2]), and require additional locking servos. The extra loss will result in only $\sim 0.1 \mathrm{~dB}$ reduction of the squeezing, while the locking of the arm cavities will be independent of the squeezing.

With regard to signal recycling, modeling of a system with long signal storage times [21] has shown that the squeezing will sense an impedance matched cavity and therefore be transmitted through the interferometer at the signal frequency. Squeezing is nonetheless advantageous as it will broaden the bandwidth of the signal recycling. With resonant sideband extraction (RSE) [23], the injected

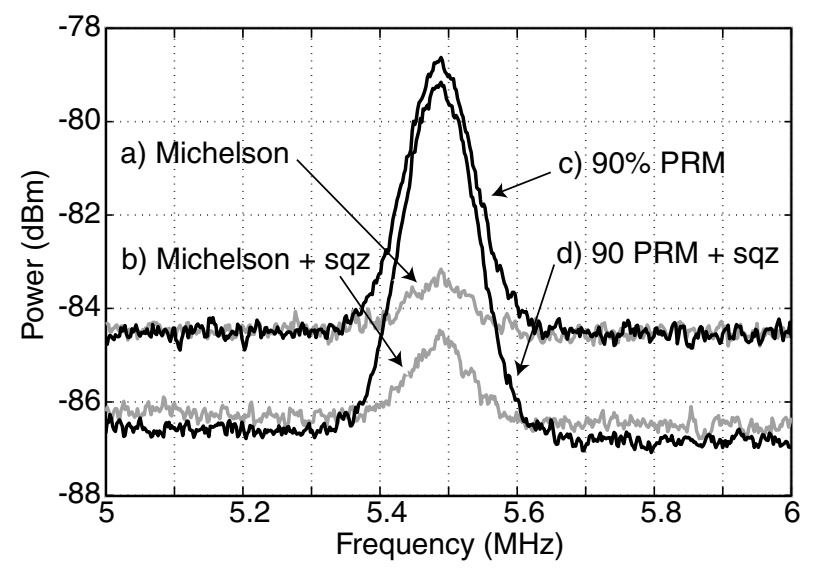

FIG. 4. Results showing subshot noise sensitivity in our interferometer, with and without power recycling. Electronic noise lies at $-93.5 \mathrm{dBm}$; the signal is at $5.46 \mathrm{MHz}$; RBW = $100 \mathrm{kHz} ; \mathrm{VBW}=30 \mathrm{~Hz}$. 
squeezing will sense a reflective cavity. In this case, we do not expect significant degradation of squeezing due to signal recycling. Employing squeezing and signal recycling together, while feasible, might not be a worthwhile exercise. Squeezing is helpful only if quantum noise sources are limiting the interferometer performance. A combination of RSE and squeezing is likely to reach a regime where other noise sources, particularly thermal noise, will be dominant at low frequencies [1]. In this case, squeezing will be of benefit only at higher frequencies, outside the thermal noise bandwidth. Instead, one may imagine a squeezed, power recycled interferometer with arm cavities as an alternative to RSE [2,24].

With respect to the squeezing bandwidth, our experiment shows squeezing at $\mathrm{MHz}$, rather than the $\sim 100 \mathrm{~Hz}$ range required for GW detection. The major impediment to benchtop low frequency squeezing is laser technical noise. The lowest squeezing frequency in a continuous wave beam reported to date is $200 \mathrm{kHz}$, which was achieved by the use of two OPAs, enabling common-mode rejection of the classical noise [25]. Fortunately, GW detection facilities provide the ideal environment for low frequency squeezed state generation. In particular, there will be a ready supply of laser light shot noise limited to frequencies as low as $10 \mathrm{~Hz}$ [19], suitable for generation of squeezing inside the $\mathrm{GW}$ detection bandwidth.

The key issue governing the amount of squeezing that can be realistically injected into a GW detector is the availability of low loss optics, both for squeezing generation and application to the interferometer. The dominant loss in our interferometer was the $15 \%$ double pass transmission of the rotator. Given the low absorption of the materials used in rotators (such as terbium gallium garnet with absorption of $0.55 \% \mathrm{~cm}^{-1}$ ), careful tuning of the magnetic fields around the crystal should provide rotators with $<5 \%$ double pass loss. The amount of squeezing generated is limited by material losses in the OPA crystal. The high laser powers available in GW facilities will allow squeezing generation in regimes not possible in the benchtop system. High laser powers may be used to pump OPA cavities with much lower finesse, thereby reducing the loss of the squeezing that occurs inside the OPA cavity. Given that benchtop squeezing experiments currently measure $7 \mathrm{~dB}$ of quantum noise suppression [7,8], over $10 \mathrm{~dB}$ of squeezing is a realistic target in a GW facility.
In summary, the technology of high precision interferometry and squeezed state generation have now reached a stage where their fusion presents a genuine alternative configuration for future gravitational wave detectors. Our results, which show the compatibility of advanced interferometer design and squeezed light, represent the first step toward this goal.

The authors acknowledge Malcolm Gray for the design of the electronics used in our experiment and the funding of the Australian Research Council.

[1] E. Gustafson et al., LIGO T990080-00-D, 1999; http://www.ligo.caltech.edu/docs/T/T990080-00.pdf

[2] H. J. Kimble et al., Phys. Rev. D 65, 022002 (2002).

[3] C. M. Caves, Phys. Rev. D 23, 1693 (1981).

[4] V. B. Braginsky et al., Phys. Rev. D 61, 044002 (2000).

[5] A. Buonanno and Y. Chen, Phys. Rev. D 64, 042006 (2001).

[6] R. E. Slusher et al., Phys. Rev. Lett. 55, 2409 (1985).

[7] K. Schneider et al., Opt. Ex. 2, 59 (1998).

[8] P. K. Lam et al., J. Opt. B 1, 469 (1999).

[9] M. Xiao et al., Phys. Rev. Lett. 59, 278 (1987).

[10] P. Grangier et al., Phys. Rev. Lett. 59, 2153 (1987).

[11] J. Gea-Banacloche and G. Leuchs, J. Mod. Opt. 34, 793 (1987).

[12] B. J. Meers, Phys. Rev. D 38, 2317 (1988).

[13] A. Abramovici et al., Science 256, 325 (1992).

[14] A. Rüdiger et al., Opt. Acta 28, 641 (1981).

[15] B. C. Buchler et al., Phys. Rev. A 65, 011803(R) (2002).

[16] A benchtop interferometer is limited to $\mathrm{MHz}$ signals due to the short power cavity. The power cavity linewidth of a long baseline interferometer will be of the order of $100 \mathrm{~Hz}$.

[17] R. W. P. Drever et al., Appl. Phys. B 31, 97 (1983).

[18] A. J. Stevenson et al., Appl. Opt. 32, 3481 (1993).

[19] K. Strain, "LIGO II Configuration Options," http://www.phys.ufl.edu/LIGO/LIGO/DOCS/config_1.pdf

[20] L. Schnupp, conference presentation, European Collaboration Meeting on Interferometric Detection of Gravitational Waves, 1988.

[21] V. J. Chickamane et al., Phys. Rev. A 57, 3898 (1998).

[22] A. F. Pace et al., Phys. Rev. A 47, 3173 (1993).

[23] J. Mizuno et al., Phys. Lett. A 175, 273 (1993).

[24] D. E. McClelland et al., in Proceedings of the Second TAMA Conference, edited by S. Kawamura and N. Mio (Academic, New York, 2000), p. 355.

[25] W. P. Bowen et al., quant-ph/0205097. 